\title{
A proposal for Climate Change Resilience Management through Fuzzy Controllers
}

\author{
J. Rubén G. Cárdenas ${ }^{1}$, Àngela Nebot ${ }^{2}$, Francisco Mugica ${ }^{2}$ \\ ${ }^{1}$ IUSS UME School, Via Ferrata 45, Pavia, Italy \\ ${ }^{2}$ Soft Computing Group, Technical University of Catalonia, Jordi Girona Salgado 1-3, Barcelona, Spain \\ ruben.gonzalez@umeschool.it,angela@lsi.upc.edu,fmugica@lsi.upc.edu
}

\begin{abstract}
Keywords: $\quad$ Fuzzy Sets, Risk Management, Natural Hazards, Social Vulnerability, Resilience Management, Fuzzy Controllers, Inference System, Decision Support System, Artificial Intelligence

Abstract: We aim towards the implementation of a set of fuzzy controllers capable to perform automated estimation of the period of time necessary to recover a resilience level through the non-linear influence of a set of interrelated climate change resilience indicators constrained by social-based variables. This fuzzy controller set, working together with a fuzzy inference system type Mamdani, will be capable to estimate the proper adjustments to be done onto system's elements in order to achieve a certain resilience level, while a general estimation of required costs is appraised. The final tool can then be used to provide guidelines for strategic vulnerability planning and monitoring through a clear understanding between investments and results, while an open evaluation and scrutiny of applied policies is made. In this paper the main strategy to achieve the mentioned objectives is presented and discussed.
\end{abstract}

\section{INTRODUCTION}

Resilience is one of the risk components that might be viewed as real interface between analysis and decisions. Moreover, resilience can be considered as a linkage between mother's Nature uncertainty and social-based structures. Such as risk, in order to enhance societies resilience capacities, a transparent and consistent decision framework must be designed while including an assertion of its capacity to be implemented over those official bodies responsible of binding either social behaviors and/or interactions. Up to some point, it is when these social institutions fails that susceptibility to external or internal stressors increases, and the whole management structure seems to be compromised. Resilience however, is a concept not completely well understood, even if its final aim is in somehow clear.

It is by correlating the concept of recovery to real factors, that a true improvement on resilience strategies assessments and implementations on a real scenario can be achieved, however the shift from qualitative resilience models towards quantitative resilience assessment models represents a very recent branch in the field of disaster risk management. Consequently, most of techniques dealing with disaster resilience modeling and assessment rely on either: probabilistic approaches (Cimellaro et al., 2010; Miles and Chang, 2006; Bruneau et al., 2003), indexing (Carreño et al., 2013; Cardona, 2001) and/or qualitative ratings (Astles et al.,2009; Hobday 2011). Even practical, these approaches do leave information aside, either by not considering system's elements interrelationships or dynamics, or because they fail to handle concepts intimately related with impreciseness and perceptions. In the same way, most of the resilience assessments models leave the temporal dimension of resilience unattended. Our purpose in this research is to achieve reliable recovery time estimations while a proper supervision and control of resilience indicators' progress is performed. Because of its simple configuration, the set of fuzzy controllers might be very helpful in provide guidelines suitable for medium and long term strategic adaptation planning since the final 'target' resilience level can be selected by the final user. From there, the resilience controller is capable to estimate the proper adjustments that needs to be done to each indicator's performance rate in order to achieve such level, while a general estimation of required costs is apprised. 


\section{MEASURING CLIMATE IMPACTS THROUGH INDICATORS}

According to Ellis (2014), there is no absolute consensus whether it is better to focus on vulnerability or resilience when using indicators to describe and explain climate impacts to communities. Still, Malone (2009) stated that although most of climate change research followed in its origins a more vulnerability focused approach, there is an ongoing slow shifting towards an adaptive resilience-based approach because the concept of resilience, often considered as an ability can be embraced as an integral part of general and particular development goals, while vulnerability alone is often assumed as a degree. Certainly, both concepts are related and someone may think that one establishes the other and vice versa, nevertheless theirs is not a causal relationship since a resilient community is still vulnerable and in any case, a state of minimum vulnerability would be a complex outcome that goes beyond of the resilience goal only.

Research on resilience indicators linked to climate change is limited, and different approaches may be used for its development. One of the most recurrent is the one that consider resilience as the opposite to vulnerability, which is defined in the context of climate change as a combination of exposure, sensitivity and adaptive capacity (IPCC, 2005). In this sense, vulnerability will be reduced through improving resilience capacities.

On the other hand, resilience operability can be considered as a matter of purely adaptive capacities. Malone (2009) defines adaptive capacity as: "the ability to design and implement effective adaptation strategies, or to react to evolving hazards and stresses so as to reduce the likelihood of the occurrence and/or the magnitude of harmful outcomes resulting from climate-related hazards."

By adopting this view it is possible to use the wide range of research developed to measure adaptive capacity to climate change, to develop resilience indicators, hence turning resilience into a more measurable entity. However, resilience and adaptive capacity are not simply interchangeable and their relationship must be established with caution. Carpenter et al., (2001) highlighted that relationship between resilience and adaptive capacity can be established in terms of the capacity of the social system to endure and overcome changes coming from external or internal forces. For Carpenter, the capacity of self-organization and learning capacity building determines the rate of change that a systems can undergo and therefore, a way to estimate resilience.
Since we intend to perform a monitoring scheme over the performance of a set of socio-economic indicators while considering the well functioning of a community official bodies to estimate the climate impacts that may occur over certain ecosystem services and socio-economic conditions, in this study we are choosing a combination of the both aforementioned resilience frameworks, that is: an adaptive resiliencebased approach to estimate the influences of coordinate official bodies, and a vulnerability approach that considers all components assumed to integrate such a concept in space and time.

\section{RESILIENCE INDICATORS}

The use of indicators to quantify an intangible concept such as resilience has always caused some controversy. In fact, the common thought now is that resilience cannot be measured directly and instead, a set of proxy variables to perform comparative monitoring and assessment over time is needed (Silver et al., 2008). In doing so, a very extended methodology to describe a complex reality in terms of indicators is the so called composite indices, which are intended to integrate trough aggregation all components described by individual indicators into a single dimensionless number or class. Despite the difference in approaches there are some common features to all of the resilience indicators considered in this study.

\subsection{Vulnerability Approach Indicators}

We intent to use resilience indicators presented by Moss et al. (2001): Ibarraran et al. (2010) in the so called Vulnerability- Resilience Indicators Model (VRIM), which focuses on sensitivity and adaptive capacity, while exposure is regarded as implicit. The VRIM model is divided in 8 different sectorial indicators obtained trough the aggregation of different proxy variables attempted to describe each sector (see Table 1). In the VRIM model, all proxy variables described by indicators are aggregated using geometric averages, after a process of normalization.

\subsection{Adaptive Capacity Approach Indicators}

In order to develop indicators under this category, a set of components or determinants of resilience specific to a particular area or sector is defined first. Focusing on urban climate resilience, Swanson et al., (2009) proposed a framework for climate resilience indicators development considering six components: 
Table 1: Vulnerability-Resilience Indicators Model (VRIM) (Moss et al. 2001, Ibarraran et al. (2010)

\begin{tabular}{|r|c|}
\hline Sectorial Indicators & Proxy Variables \\
\hline Food Security & $\begin{array}{c}\text { Cereals production/ crop land area } \\
\text { Protein consumption/ capita }\end{array}$ \\
\hline Water resource sensitivity & Renewable supply and inflow of water \\
\hline Settlement / infrastructure sensitivity & $\begin{array}{c}\text { Population at flood risk from sea level rise } \\
\text { Population without access to clean water } \\
\text { Population without access to sanitation }\end{array}$ \\
\hline Human health sensitivity & Completed fertility \\
& Life expectancy \\
\hline Ecosystem sensitivity & $\%$ Land managed \\
& $\%$ Fertilizer use / cropland area \\
\hline Human and civic resources & Dependency ratio \\
& Literacy \\
\hline Economic capacity & GDP(market) / capita \\
& An income equity measure \\
\hline Environmental capacity & Land unmanaged \\
& SO ${ }_{2} /$ area \\
& Population density \\
\hline
\end{tabular}

- Economic resources:

- Technology

- Information, skills and management

- Infrastructure

- Institutions and networks

- Equity

We are now in the process of selecting the proper set of sectors to cover all these dimensions.

\section{APPROACH}

The proposed strategy is divided in two main stages, each on one involving different activities. The first part of the strategy comprises the development and implementation of the Climate Resilience Fuzzy Inference model that will be used to estimate resilience levels, while the second part comprise the construction and implementation of the Climate Resilience Fuzzy Controller that will be used to estimate resilience recovery times. Figure 1 shows a conceptual diagram of the main functioning of the proposed modeling scheme.

\subsection{Development of the Resilience Fuzzy Inference model}

Classical inferences are based on tautologies given by propositional calculus, which aim is the study of propositions formed by logical connectors. Through the concept of formal system, propositional logic represents propositions by means of formulas depicting natural language that can be used to form rules of inferences. A rule of inference is then a function that considers premises and, by analyzing their syntax, returns a conclusion. Classical rules of inference can be generalized for its implementation in the context of fuzzy logic theory through the use of linguistic variables expressed as quantitative terms, and compositional rules of inference. Ultimately, these two elements state the framework that holds the field of approximate reasoning.

A fuzzy expert system tries to emulate the reasoning process of a human expert within a specific domain of knowledge; an expert system is then a fuzzy inference system if the reasoning process is made over a data set by means of fuzzy rules, which expresses the actual knowledge about a particular problem.

We are following the fundamental steps required by an expert fuzzy system to be implemented. Mainly:

Step 1. Build a knowledge base, which is represented by a set of fuzzy rules if-then type interrelat- 


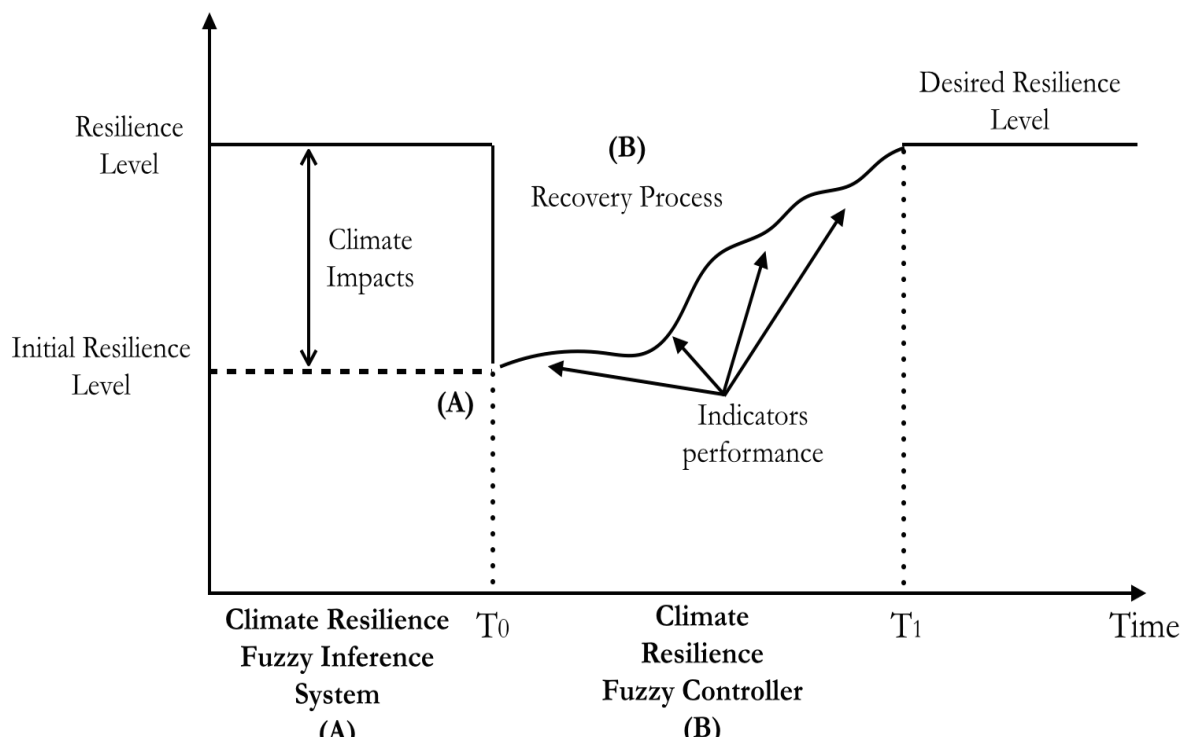

(A)

(B)

Figure 1: Conceptualization of the proposed modeling scheme (A) A resilience level is estimated by the Climate Fuzzy Inference System. (B) Control actions are taken trough the implementation of the Climate Resilience Fuzzy Controller onto indicators' performance.

ing each selected resilience indicator in order to reflect our knowledge about climate resilience formation and evolution. In this way, we would be capable to assemble compositional rules of inference over the very same indicators that are assumed to create a certain resilience level. Our main sources of information would be based on a detailed resilience literature review and subjective assessments coming from international experts in the field, which in turn can be quantified by means of participatory questionnaires properly pondered by Delphi or Hierarchical Analytic Process techniques (Saaty, et al, 1991). By following this scheme, we want to induce a multidisciplinary discussion among climate and social science scholars, in order to improve rule's structure strength.

Step 2. In order to fuzzify the reported indicator's values, as well as their possible outcomes after the inference, a database of fuzzy sets must be defined in terms of membership functions and a definition of meaningful linguistic states for each indicator. Possible membership functions shapes and their respective universes of discourses would be consolidated, as stated in the previous point, by subjective assessments coming from international experts in the field. Our goal is to achieve a reliable membership functions database considering a different range of shapes to be implemented on the FIS model.

Step 3. In the fuzzification module, original data will be converted into fuzzy sets by determining the match between the input raw indicator data and its de- fined fuzzy set (membership function). These fuzzified values will be used to evaluate those rules presented in the knowledge base.

Step 4. In the model's inference engine, each fuzzy rule stored in the knowledge base will be evaluated in order to perform fuzzy inferences. In order to evaluate each rule, the fuzzy inference engine determines a firing strength for each rule considering the degree of match and the fuzzy connectors used over antecedents. Afterwards, an estimation of the outputs is made based on the estimated fire strength and the match with the defined fuzzy sets for output variables in the consequent part of each rule. Once rules have been evaluated, an aggregation process would be made through a fuzzy aggregator operator.

Step 5. In the defuzzification module of the model, the inference result or the aggregate outcome (a fuzzy set), would be re-converted by means of a defuzzification process into a crisp, scalar value; weather to obtain a final estimation or for further processing.

\subsection{Development of the Resilience Fuzzy Controller}

The general aim of a fuzzy controller is to process fuzzy and non fuzzy information in a fuzzy scheme of reasoning in order to determine if the current system utility performance is in line with predetermined standards and if necessary, to take remedial actions over system's variables in order to assure that they 


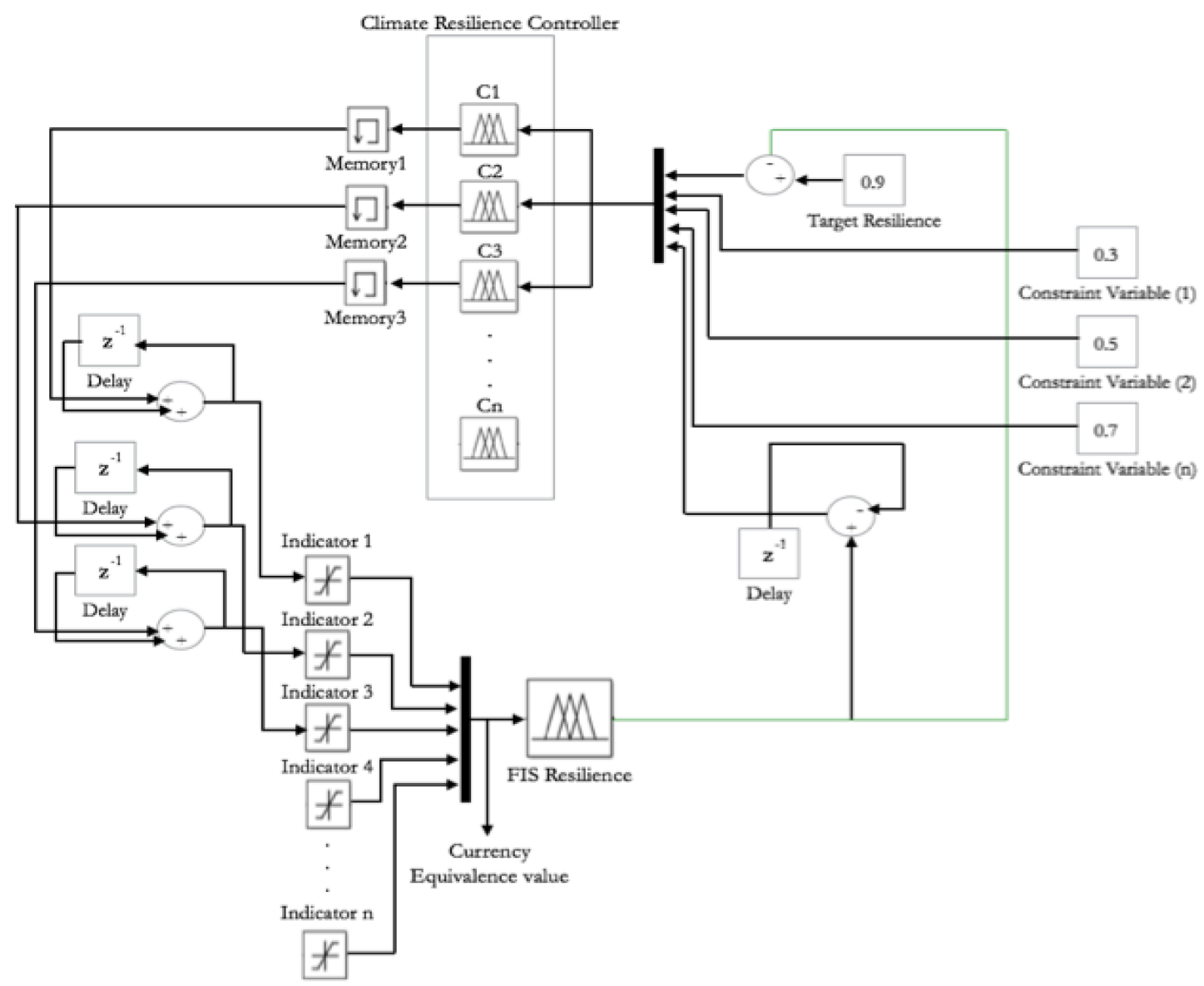

Figure 2: Conceptualization of the resilience fuzzy controller closed-loop operation. Once a resilience level is estimated by a FIS by means of a non-linear aggregation of a set of $n$ resilience indicators, input control variables are selected (such as the resilience target level, and the values for constrained variables). Then a subset of fuzzy controllers $C_{i}$ will be activated depending on the relevance of their related indicator for the considered climate impact and its capacity to be managed through control actions (As an example, in the figure the selected indicators are: 1, 2, 3, and the activated fuzzy controllers are: $\mathrm{C} 1, \mathrm{C} 2, \mathrm{C} 3$ respectively. The rest of indicators might be considered as constants or to be associated to a rate of change on their own). These selected controllers will perform control actions over the action rates of their respective resilience indicators in order to achieve the target resilience level. Before each cycle starts again, an exact appraisal on the investment cost needed to achieve that particular resilience level will be estimated.

are being used in the most effective way in achieving planned objectives. The proposed Resilience Controller will use two system's states: a desired resilience level that must be selected by the final user, and an observed/measured resilience state, which will be estimated in advance by the Climate Resilience Fuzzy Inference model. The fuzzy controller works through comparing the desired resilience state to the estimated resilience state and then adjusting the necessary variables by considering the difference between the two mentioned resilience levels and an a specified control strategy.

The input variables of the fuzzy controller will be the outputs of the Resilience Fuzzy Inference model, which are already fuzzy sets. In this scheme we're proposing, an important feature comes with those variables on which control actions are going to be taken (control-output variables) which are those of the resilience indicators already defined in the Climate Resilience Fuzzy Inference model on which control actions can be managed. Each of these indicators should have associated a particular ratio value, for example: number of physicians reported per year, $\mathrm{CO} 2$ production per year, percentage of population with higher education reported per year, unemployment rate per year, etc. It is through these temporal snapshots that the so-called action range pertaining to each resilience indicator can be established and then, control actions can be designed. The domains of all control variables will be divided in fuzzy sets, which 
allow their handling in a linguistic manner.

By following this scheme we are ensuring that the controller remains engaged to a qualitative scale representing only realistic rates of change that can be achieved by each indicator per unit of time. Moreover, the same rate can be used as an alternative scale in terms of monetary value, since all ratio values can be translated to production costs and therefore, all estimations made by the fuzzy controller can be easily translated into currency investments.

The Climate Resilience Fuzzy Controller will use fuzzy logic rules to relate input variables to those changes that would need to be made over resilience indicators in order to achieve a particular resilience level. Controller's rules are in some how different from the previous mentioned fuzzy inference rules since the aim now is to design a controlled performance over output variables. Therefore the main structure of a control rule relates conditions to control actions, in this sense if conditions are in the form of linguistic variables representing two process state variables, a control rule implemented by a fuzzy implication, will lead into an action over control variables.

As we mentioned before, once the changes over output variables have been established, they will be used again as inputs for the aforementioned Climate Resilience Fuzzy Inference model in order to achieve final resilience estimations. Working together, the Fuzzy Inference model and the Resilience Fuzzy Controller can establish a loop-based framework suitable to perform resilience estimations while maintaining control actions over indicators performance. It is important to mention that although each indicator aims to describe a particular system's feature or condition, not all of these features will be controllable and in any case, the relevance of each indicator to influence a potential resilience level will be related with the type of climate impact under scope (whether droughts, floods, etc.) Therefore the proposed controlling scheme will be based on a careful selection of which variables/indicators are potentially controllable and may influence as well a possible outcome. For example, in a drought event, the control actions would be related with indicators depicting food security and water resources, while in the case of a climate impact in the form of floods, the settlement / infrastructure sensitivity indicator would be more suitable to be used as a guide to implement control actions.

Once the Fuzzy Resilience model has been completed, we intent to implement it over at least two cities by following a scenario-building approach. Therefore, in order to define a set of different scenarios we will use explicits combinations of values belonging to constrains variables, along with the potential responses of official bodies in terms of: budget allocation and the political willingness to embrace and implement adaptation-resilience strategies. Others types of variables, not described by the aforementioned set of indicators but critical to describe particular social conditions (such as: social development, risk perceptions, learning capacities, communication between stakeholders, etc.) can be included in this stage.

In this way, applying the final resilience controller would consist in first defining a particular target resilience level and then selecting those input parameters aimed to describe a particular user's condition, either social or political. The target resilience level would be completely configurable and will be depending on those particular goals requested by the final user. Since the time lapse to achieve this level depends entirely on real indicator's rates of changes, and a direct cost of the experiment can be automatically obtained, a final user could re-configure if needed, whether input parameters or the final resilience level in order to be consequent with his own resources and or particular resilience strategic planning.

The whole scheme of the planned Resilience Inference Model, working together with the set of Fuzzy Controllers can be seen in Figure 2.

\section{CONCLUSIONS}

In this study, we propose a decision making assistance tool in the form of fuzzy controllers. This tool will estimate the amount of time needed to recover a certain resilience level related to the actions of climate impacts and global change while considering a coerced social and political landscape. We propose the use of climate resilience indicators, vastly reported in literature. By following a resilience framework aimed to measure both, vulnerability and adaptive capacities, our intention is to estimate first an initial resilience level by means of fuzzy composite indices, where indicators aggregation is made assuming a non-linear interdependence among selected indicators, contrary to more traditional composite indices methodologies. This resilience level can then be used as initial input of a set of fuzzy controllers that implements control actions over indicators' performance ratios in order to achieve a resilience level that can be selected by the final user. At the same time that the so called resilience target level is achieved, an estimation of the required investment costs to achieve such level is calculated, considering the rate of production reported for each indicator. 
Resilience fuzzy controller's configuration options allows manageable projections considering diverse circumstances that might be very helpful to answer questions such as: if a certain climate resilience level is required, what are those achievable changes that needs to be done onto climate resilience indicators? Or: in how long these changes would be reflected in a real resilience improvement?

Finally, and since the Climate Resilience fuzzy controller comprise just an element of the integral risk to global change, an immediate future extension of our current work would involve the development of a full climate risk fuzzy controller.

\section{References}

Astles K. L., Gibbs P. J., Steffe A. S., Green M. (2009) A qualitative risk-based assessment of impacts on marine habitats and harvested species for a data deficient wild capture fishery. Biological Conservation ,142, 2759-2773.

Bruneau, M., and others (2003), A framework to quantitatively assess and enhance the seismic resilience of communities, Earthquake Spectra, 19(4) 733-752.

Carreño, M.L., Cardona, O.D., Barbat, A.H., (2012), New methodology for urban seismic risk assessment from a holistic perspective, Bull Earthquake Eng,10, 47-565.

Cardona, O.D., (2001) Holistic evaluation of the seismic risk using complex dynamic systems (in Spanish), PhD Thesis Technical University of Catalonia, 10, 47-565.

Carpenter, S., B. Walker, J. M. Anderies and N. Abel (2001) From Metaphor to Measurement: Resilience of What to What? Ecosystems, ,4(8), 765-781.

Hobday AJ, Lough J (2011) Projected climate change in Australian marine and freshwater environments. Mar FreshwRes,62 1000-1014.

IPCC, (2005), IPCC Special Report on Carbon Dioxide Capture and Storage. Prepared by Working Group III of the Intergovernmental Panel on Climate Change, Cambridge University Press 12(3) 442.

Ibarraran ME, Malone EL, Brenkert AL. (2010), Climate Change Vulnerability and Resilience: Current Status and Trends for Mexico, Environment, Development and Sustainability, 12(3) 365.

Jennifer Ellis, (2014) Climate Resilience Indicator Literature Review. Trust's Communities Adapting to Climate Change Initiative and the Columbia Basin Rural Development Institute, Columbia Basin Trust.

Miles, S.B., and S.E. Chang (2006), Modeling community recovery from earthquakes, Earthquake Spectra,
22(2) $439-458$

Mamdani, E.H., Assilian, S., (1975) An experiment in linguistic synthesis with a fuzzy logic controller. Intern. J. of Man-Machine Studies,7(1), 1-13.

Malone, E.L. (2009). Vulnerability and Resilience in the Face of Climate Change: Current Research and Needs for Population Information, Population Action International: Washington DC., www.populationaction.org

Moss RH, Brenkert AL, Malone EL. (2001), Vulnerability to Climate Change: A Quantitative Approach, Pacific Northwest National Laboratory PNNL-SA-33642.

T.L. Saaty, L.G. Vargas. (1991), Prediction, Projection, and Forecasting: Applications of the Analytical Hierarchy Process in Economics, Finance, Politics, Games, and Sports, Kluwer Academic Publishers, Boston, MA. 